\title{
Linear and Nonlinear Optical Spectroscopy of Fluoroalkylated BODIPY Dyes
}

Dominik K. Kölmel, ${ }^{\dagger}$ Anna Hörner, ${ }^{\dagger}, \neq, \mid$ Juan A. Castañeda, ${ }^{\ddagger}$ Julio A. P. Ferencz, ${ }^{\ddagger}$ Angela Bihlmeier," Martin Nieger, ${ }^{\S}$ Stefan Bräse, ${ }^{*,+, \|}$ and Lazaro A. Padilha*,‡

†Institute of Organic Chemistry, Karlsruhe Institute of Technology, 76131 Karlsruhe, Germany

‡Instituto de Fisica “Gleb Wataghin”, Universidade Estadual de Campinas, C. P. 6165, 13083-970 Campinas, Sao Paulo, Brazil

ILight Technology Institute, Karlsruhe Institute of Technology, 76131 Karlsruhe, Germany

"Institute of Physical Chemistry, Karlsruhe Institute of Technology, 76131 Karlsruhe, Germany

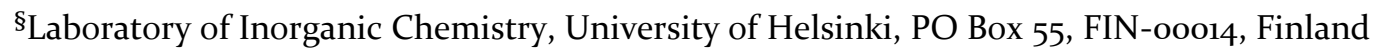

"Institute of Toxicology and Genetics, Karlsruhe Institute of Technology, 76344 EggensteinLeopoldshafen, Germany

\section{Table of content}

\section{General Information}

2. Experimental Procedures and Characterization

3. Computational Studies

4. Lifetime Fittings

5. Nonlinear Spectroscopy

6. Crystallographic Data

7. References 


\section{General Information}

All chemicals were used as received. All reactions were carried out under stirring. Reactions under inert gas were carried out in flasks equipped with septa under argon (supplied by using a standard manifold with vacuum and argon lines). Analytical TLC was performed on MERCK readyto-use plates with silica gel 60 (F254). MERCK silica gel 60 (0.04-0.063 mm) was used for column chromatography. IR spectra were recorded by using FT-IR Bruker ALPHA-T spectrometer. The samples were measured by using the attenuated total reflexion (ATR) technique. The transmission intensities of the bands were characterized as follows: $\mathrm{s}=$ strong $(11-40 \%), \mathrm{m}=$ medium $(41-70 \%)$, $\mathrm{w}=$ weak $(71-90 \%), \mathrm{vw}=$ very weak $(91-100 \%)$ transmission. NMR spectra were recorded at $25{ }^{\circ} \mathrm{C}$ by using Bruker AM $400\left(400 \mathrm{MHz}\left({ }^{1} \mathrm{H}\right), 376.5 \mathrm{MHz}\left({ }^{19} \mathrm{~F}\right)\right.$ and $\left.100 \mathrm{MHz}\left({ }^{13} \mathrm{C}\right)\right)$ and Bruker DRX 500 $\left(500 \mathrm{MHz}\left({ }^{1} \mathrm{H}\right)\right)$ spectrometer. All spectra are referenced to tetramethylsilane as standard $(\delta=0 \mathrm{ppm})$ by using the signals of the solvent:

\section{$\mathrm{CDCl}_{3}: 7.26 \mathrm{ppm}\left(\mathrm{CHCl}_{3}\right)$ or $77.16 \mathrm{ppm}\left({ }^{13} \mathrm{CDCl}_{3}\right)$}

\section{$\mathrm{CD}_{3} \mathrm{CN}: 1.94 \mathrm{ppm}\left(\mathrm{CHD}_{2} \mathrm{CN}\right)$ or 1.32 and $118.26 \mathrm{ppm}\left({ }^{13} \mathrm{CD}_{3} \mathrm{CN}\right.$ and $\left.\mathrm{CD}_{3}{ }^{13} \mathrm{CN}\right)$}

$\mathrm{d}_{6}$-acetone: $2.05 \mathrm{ppm}\left(\mathrm{CHD}_{2} \mathrm{COCD}_{3}\right)$ or 29.84 and $206.26 \mathrm{ppm}\left({ }^{13} \mathrm{CD}_{3} \mathrm{COCD}_{3}\right.$ and $\left.\left(\mathrm{CD}_{3}\right)_{2}{ }^{13} \mathrm{CO}\right)$

\section{$\mathrm{d}_{8}$-THF: 1.73 and $3.58 \mathrm{ppm}\left(\mathrm{C}_{4} \mathrm{HD}_{7} \mathrm{O}\right)$}

The spectra were analyzed according to first order. Multiplicities of the signals are described as follows: $\mathrm{s}=$ singlet, $\mathrm{bs}=$ broad singlet, $\mathrm{d}=$ doublet, $\mathrm{t}=$ triplet, $\mathrm{q}=$ quartet, $\mathrm{dd}=$ doublet of doublet, $\mathrm{dt}=$ doublet of triplet, $\mathrm{m}=$ multiplet. Coupling constants $(J)$ are given in Hz. Multiplicities in the ${ }^{13} \mathrm{C}$ NMR spectra were determined by DEPT (distortionless enhancement by polarization transfer) measurements. Perfluorinated carbon atoms (with the exception of the perfluoro-tert-butyl moiety) and carbon atoms directly bound to boron were not analyzed by ${ }^{13} \mathrm{C}$ NMR spectroscopy due to their weak signals. Mass spectra (EI and FAB) were obtained by using a Finnigan MAT 90 spectrometer. MALDI-TOF mass spectra were obtained by using a Bruker Biflex IV spectrometer with a pulsed ultraviolet nitrogen laser, $200 \mu \mathrm{J}$ at $337 \mathrm{~nm}$ and a time-of-flight mass analyzer with a $125 \mathrm{~cm}$ linear flight path. A 1:1 mixture of 2,5-dihydroxybenzoic acid and $\alpha$-cyano-4-hydroxycinnamic acid (dissolved in $\mathrm{H}_{2} \mathrm{O}$ /acetonitrile (1:1) with $0.1 \%$ TFA) was used as matrix.

UV/Vis absorption spectra were recorded by using a Varian Cary 300 UV Visible spectrophotometer and fluorescence spectra were recorded by using a Varian Cary Eclipse fluorescence spectrophotometer. Closed quartz cuvettes with a $1 \mathrm{~cm}$ path length were used in all experiments. 
Fluorescence quantum yield measurements were performed on the previously mentioned fluorometer and UV/Vis instrument. The slit width was $5 \mathrm{~nm}$ for both excitation and emission. Relative quantum efficiencies were obtained by comparing the absorption values and the areas under the emission spectrum for the unkown substance and a standard. ${ }^{1}$ The following equation was used to calculate quantum yields:

$$
\Phi_{\mathrm{x}}=\Phi_{\mathrm{s}} \times\left(F_{\mathrm{x}} / F_{\mathrm{s}}\right) \times\left(n_{\mathrm{x}} / n_{\mathrm{s}}\right)^{2} \times\left(A_{\mathrm{s}} / A_{\mathrm{x}}\right)
$$

Here, $\Phi_{\mathrm{s}}$ is the reported quantum yield of the standard, $F$ is the integrated emission spectrum, $A$ is the absorbance at the extinction wavelength, and $n$ is the refractive index of the solvents used. The subscript $\mathrm{x}$ denotes unkown and s denotes standard. 5(6)-Carboxyfluorescein in $0.1 \mathrm{M}$ aqueous $\mathrm{NaOH}$ ( $\Phi_{\mathrm{F}}=0.95$; for dye 1$)$, Atto 532 in water $\left(\Phi_{\mathrm{F}}=0.90\right.$; for dye 2$)$, or rhodamine 101 in methanol $\left(\Phi_{\mathrm{F}}=0.99\right.$; for dye $\left.\mathbf{4}-\mathbf{8}, \mathbf{1 0}\right)$ were used as standards.

\section{Experimental Procedures and Characterization}

\section{4,4-Difluoro-1,3,5,7-tetramethyl-8-(perfluorophenyl)-4-bora-3a,4a-diaza-s-indacene (1):}

The preparation and properties of compound $\mathbf{1}$ have been reported in reference 2 .

\section{4,4-Difluoro-2,6-diiodo-1,3,5,7-tetramethyl-8-(perfluorophenyl)-4-bora-3a,4a-diaza-s- indacene (2):}

BODIPY dye $1(150 \mathrm{mg}, 362 \mu \mathrm{mol})$ and iodine $(230 \mathrm{mg}, 905 \mu \mathrm{mol})$ were dissolved in $38 \mathrm{~mL}$ ethanol. To this solution iodic acid (127 mg, $724 \mu \mathrm{mol})$, dissolved in $300 \mu \mathrm{L}$ water, was added and stirred overnight at $60{ }^{\circ} \mathrm{C}$. After cooling to ambient temperature, $10 \mathrm{~mL}$ saturated aqueous $\mathrm{Na}_{2} \mathrm{~S}_{2} \mathrm{O}_{3}$ solution, $30 \mathrm{~mL}$ water and $80 \mathrm{~mL}$ dichloromethane was added. The organic layer was separated, washed with $30 \mathrm{~mL}$ water and evaporated under reduced pressure. The crude product was purified by using column chromatography (eluent cyclohexane/ $\mathrm{CH}_{2} \mathrm{Cl}_{2}$ 5:1) to give a red solid (207 mg, 86\%).

M.p. $210{ }^{\circ} \mathrm{C}$ (decomposition); ${ }^{1} \mathrm{H}$ NMR (400 MHz, $\left.\mathrm{CDCl}_{3}\right): \delta=1.64(\mathrm{~s}, 6 \mathrm{H}), 2.67(\mathrm{~s}, 6 \mathrm{H}) ;{ }^{13} \mathrm{C}$ NMR (100 MHz, $\left.\mathrm{CDCl}_{3}\right): \delta=16.2\left(\mathrm{CH}_{3}\right), 16.3\left(\mathrm{CH}_{3}\right), 87.0\left(\mathrm{C}_{\mathrm{ar}}\right), 122.5\left(\mathrm{C}_{\mathrm{ar}}\right), 130.9\left(\mathrm{C}_{\mathrm{ar}}\right), 143.7$ $\left(\mathrm{C}_{\mathrm{ar}}\right), 159.1\left(\mathrm{C}_{\mathrm{ar}}\right) ;{ }^{19} \mathrm{~F}$ NMR $\left(376.5 \mathrm{MHz}, \mathrm{CDCl}_{3}\right): \delta=-158.5\left(\mathrm{~m}, 2 \times \mathrm{CF}_{\mathrm{ar}}\right),-149.0(\mathrm{t}, J(\mathrm{~F}, \mathrm{~F})=$ 
$\left.21 \mathrm{~Hz}, \mathrm{CF}_{\mathrm{ar}}\right),-145.5\left(\mathrm{q}, J(\mathrm{~F}, \mathrm{~B})=31 \mathrm{~Hz}, \mathrm{BF}_{2}\right),-138.9(\mathrm{dd}, J(\mathrm{~F}, \mathrm{~F})=22 \mathrm{~Hz}, J(\mathrm{~F}, \mathrm{~F})=7 \mathrm{~Hz}, 2 \times$ $\left.\mathrm{CF}_{\mathrm{ar}}\right)$; FAB MS $m / z(\%): 666(100)\left[\mathrm{M}^{+}\right], 647(23)\left[(\mathrm{M}-\mathrm{F})^{+}\right]$; HR FAB MS: $m / z$ calculated for $\left[\mathrm{C}_{19} \mathrm{H}_{12} \mathrm{BF}_{7} \mathrm{I}_{2} \mathrm{~N}_{2}\right]^{+}:$665.9071, found: 665.9069; IR (ATR): $\tilde{v}\left(\mathrm{~cm}^{-1}\right)=1656(\mathrm{vw}), 1531(\mathrm{~m}), 1501$ (m), 1397 (w), 1342 (w), 1299 (m), 1210 (vw), 1164 (m), 1112 (w), 1090 (m), 1024 (w), 984 (m), $909(\mathrm{w}), 857(\mathrm{w}), 770(\mathrm{w}), 711(\mathrm{w}), 629(\mathrm{w}), 568(\mathrm{w}), 521(\mathrm{~m}), 474(\mathrm{w}), 425(\mathrm{w})$.

\section{(E)-(4-(1H,2H-Perfluorooct-1-en-1-yl)phenyl)boronic acid MIDA ester (3):}

4-Bromophenylboronic acid MIDA ester (100 mg, $321 \mu \mathrm{mol})$, Herrmann-Beller catalyst (3 mg, $3.2 \mu \mathrm{mol})$ and sodium acetate $(32.9 \mathrm{mg}, 401 \mu \mathrm{mol})$ were added to a vial. The vial was capped with a rubber septum and then purged with argon. Afterwards $1.5 \mathrm{~mL}$ dry DMF and $1 \mathrm{H}, 1 \mathrm{H}, 2 \mathrm{H}$ perfluoro-1-octene $(91.3 \mu \mathrm{L}, 401 \mu \mathrm{mol})$ were added via a syringe and the mixture was stirred for $24 \mathrm{~h}$ at $125{ }^{\circ} \mathrm{C}$. After cooling to ambient temperature, the mixture was partitioned between 10 $\mathrm{mL}$ ethyl acetate and $10 \mathrm{~mL}$ water. The organic layer was separated, washed with $2 \times 10 \mathrm{~mL}$ water, dried over $\mathrm{Na}_{2} \mathrm{SO}_{4}$ and evaporated under reduced pressure. The crude product was purified by using column chromatography (eluent cyclohexane/EtOAc 1:1 to EtOAc) to give an off-white solid (132 mg, 71\%).

M.p. $198{ }^{\circ} \mathrm{C} ;{ }^{1} \mathrm{H}$ NMR $\left(400 \mathrm{MHz}, \mathrm{CD}_{3} \mathrm{CN}\right): \delta=2.51(\mathrm{~s}, 3 \mathrm{H}), 3.90(\mathrm{~d}, J(\mathrm{H}, \mathrm{H})=17.1 \mathrm{~Hz}, 2 \mathrm{H})$, $4.08(\mathrm{~d}, J(\mathrm{H}, \mathrm{H})=17.1 \mathrm{~Hz}, 2 \mathrm{H}), 6.49(\mathrm{dt}, J(\mathrm{H}, \mathrm{H})=16.2 \mathrm{~Hz}, J(\mathrm{H}, \mathrm{F})=12.6 \mathrm{~Hz}, 1 \mathrm{H}), 7.33(\mathrm{dt}$, $J(\mathrm{H}, \mathrm{H})=16.2 \mathrm{~Hz}, J(\mathrm{H}, \mathrm{F})=2.3 \mathrm{~Hz}, 1 \mathrm{H}), 7.56(\mathrm{~d}, J(\mathrm{H}, \mathrm{H})=8.1 \mathrm{~Hz}, 2 \mathrm{H}), 7.61(\mathrm{~d}, J(\mathrm{H}, \mathrm{H})=8.1 \mathrm{~Hz}$, $2 \mathrm{H}) ;{ }^{13} \mathrm{C} \mathrm{NMR}\left(100 \mathrm{MHz}, \mathrm{CD}_{3} \mathrm{CN}\right): \delta=48.5\left(\mathrm{CH}_{3}\right), 62.8\left(\mathrm{CH}_{2}\right), 114.9(\mathrm{t}, J(\mathrm{C}, \mathrm{F})=23 \mathrm{~Hz}, \mathrm{CH})$, $128.2\left(\mathrm{CH}_{\mathrm{ar}}\right), 134.1\left(\mathrm{CH}_{\mathrm{ar}}\right), 135.2\left(\mathrm{C}_{\mathrm{ar}}\right), 141.2(\mathrm{t}, J(\mathrm{C}, \mathrm{F})=9 \mathrm{~Hz}, \mathrm{CH}), 169.4(\mathrm{C}) ;{ }^{19} \mathrm{~F}$ NMR $(376.5$ $\left.\mathrm{MHz}, \mathrm{CD}_{3} \mathrm{CN}\right): \delta=-126.6\left(\mathrm{~m}, \mathrm{CF}_{2}\right),-123.7\left(\mathrm{~m}, \mathrm{CF}_{2}\right),-123.3\left(\mathrm{~m}, \mathrm{CF}_{2}\right),-122.1\left(\mathrm{~m}, \mathrm{CF}_{2}\right),-111.3$ $\left(\mathrm{t}, J(\mathrm{~F}, \mathrm{~F})=12.8 \mathrm{~Hz}, \mathrm{CF}_{2}\right),-81.5\left(\mathrm{t}, J(\mathrm{~F}, \mathrm{~F})=10.2 \mathrm{~Hz}, \mathrm{CF}_{3}\right) ; \mathrm{FAB} \mathrm{MS} \mathrm{m} / z(\%): 578(100)$ $\left[(\mathrm{M}+\mathrm{H})^{+}\right]$; HR FAB MS: $m / z$ calculated for $\left[\mathrm{C}_{19} \mathrm{H}_{14} \mathrm{BF}_{13} \mathrm{NO}_{4}\right]^{+}:$578.0808, found: 578.0811; IR (ATR): $\tilde{v}\left(\mathrm{~cm}^{-1}\right)=3014(\mathrm{vw}), 2960(\mathrm{vw}), 1768$ (m), 1657 (w), 1609 (vw), 1458 (w), 1404 (vw), 1335 (w), 1287 (m), 1191 (s), 1142 (s), 1120 (m), 1035 (s), 992 (m), 889 (w), 851 (s), 809 (m), $735(\mathrm{~m}), 707(\mathrm{~m}), 693(\mathrm{~m}), 659(\mathrm{~m}), 636(\mathrm{w}), 567(\mathrm{w}), 530(\mathrm{~m}), 498(\mathrm{w}), 458(\mathrm{w})$.

\section{4,4-Difluoro-1,3,5,7-tetramethyl-2,6-bis(4-((E)-1H,2H-perfluorooct-1-en-1-yl)phenyl)-8- (perfluorophenyl)-4-bora-3a,4a-diaza-s-indacene (4):}

BODIPY dye 2 (30.0 mg, 45.1 $\mu \mathrm{mol}$ ), fluorous boronic acid MIDA ester 3 (57.2 mg, $99.2 \mu \mathrm{mol})$ and $\mathrm{Pd}\left(\mathrm{PPh}_{3}\right)_{4}(5.2 \mathrm{mg}, 4.51 \mu \mathrm{mol})$ were added to a vial. The vial was capped with a rubber 
septum and then purged with argon. Afterwards $900 \mu \mathrm{L}$ toluene (degassed by bubbling with argon for $30 \mathrm{~min}$ ) was added via a syringe and stirred for $10 \mathrm{~min}$ at room temperature. Then, $245 \mu \mathrm{L}$ aq. $\mathrm{NaOH}(3.0 \mathrm{M}$, degassed by bubbling with argon for $30 \mathrm{~min}$ ) was added and the mixture was stirred overnight at $80{ }^{\circ} \mathrm{C}$. After cooling to ambient temperature, the mixture was partitioned between $15 \mathrm{~mL}$ dichloromethane and $5 \mathrm{~mL}$ water. The organic layer was separated and evaporated under reduced pressure. The crude product was purified by using column chromatography (eluent cyclohexane $/ \mathrm{CH}_{2} \mathrm{Cl}_{2}$ 7:1) to give a red solid (39.2 $\mathrm{mg}, 69 \%$ ).

M.p. $184{ }^{\circ} \mathrm{C} ;{ }^{1} \mathrm{H}$ NMR $\left(400 \mathrm{MHz}, \mathrm{CDCl}_{3}\right): \delta=1.56(\mathrm{~s}, 6 \mathrm{H}), 2.56(\mathrm{~s}, 6 \mathrm{H}), 6.25(\mathrm{dt}, J(\mathrm{H}, \mathrm{H})=16.0$ $\mathrm{Hz}, J(\mathrm{H}, \mathrm{F})=12.2 \mathrm{~Hz}, 2 \mathrm{H}), 7.21(\mathrm{~d}, J(\mathrm{H}, \mathrm{H})=16.0 \mathrm{~Hz}, 2 \mathrm{H}), 7.25(\mathrm{~d}, J(\mathrm{H}, \mathrm{H})=8.2 \mathrm{~Hz}, 4 \mathrm{H}), 7.56$ $(\mathrm{d}, J(\mathrm{H}, \mathrm{H})=8.2 \mathrm{~Hz}, 4 \mathrm{H}) ;{ }^{13} \mathrm{C} \mathrm{NMR}\left(100 \mathrm{MHz}, \mathrm{CDCl}_{3}\right): \delta=11.9\left(\mathrm{CH}_{3}\right), 13.6\left(\mathrm{CH}_{3}\right), 114.8(\mathrm{t}$, $J(\mathrm{C}, \mathrm{F})=23 \mathrm{~Hz}, \mathrm{CH}), 123.5(\mathrm{C}), 127.9(\mathrm{CH}), 130.7(\mathrm{CH}), 131.1(\mathrm{C}), 132.8(\mathrm{C}), 134.0(\mathrm{C}), 134.9$ (C), $137.6(\mathrm{C}), 139.1(\mathrm{t}, J(\mathrm{C}, \mathrm{F})=9 \mathrm{~Hz}, \mathrm{CH}), 156.7(\mathrm{C}) ;{ }^{19} \mathrm{~F}$ NMR $\left(376.5 \mathrm{MHz}, \mathrm{CDCl}_{3}\right): \delta=-$ $158.9\left(\mathrm{td}, J(\mathrm{~F}, \mathrm{~F})=22 \mathrm{~Hz}, J(\mathrm{~F}, \mathrm{~F})=7 \mathrm{~Hz}, 2 \times \mathrm{CF}_{\mathrm{ar}}\right),-149.7\left(\mathrm{t}, J(\mathrm{~F}, \mathrm{~F})=21.1 \mathrm{~Hz}, \mathrm{CF}_{\mathrm{ar}}\right)$, $-145.8\left(\mathrm{q}, J(\mathrm{~F}, \mathrm{~B})=32 \mathrm{~Hz}, \mathrm{BF}_{2}\right),-139.0\left(\mathrm{dd}, J(\mathrm{~F}, \mathrm{~F})=22 \mathrm{~Hz}, J(\mathrm{~F}, \mathrm{~F})=7 \mathrm{~Hz}, 2 \times \mathrm{CF}_{\mathrm{ar}}\right),-126.1(\mathrm{~m}$, $\left.2 \times \mathrm{CF}_{2}\right),-123.1\left(\mathrm{~m}, 2 \times \mathrm{CF}_{2}\right),-122.8\left(\mathrm{~m}, 2 \times \mathrm{CF}_{2}\right),-121.5\left(\mathrm{~m}, 2 \times \mathrm{CF}_{2}\right),-111.4(\mathrm{t}, J(\mathrm{~F}, \mathrm{~F})=12$ $\left.\mathrm{Hz}, 2 \times \mathrm{CF}_{2}\right),-80.7\left(\mathrm{t}, J(\mathrm{~F}, \mathrm{~F})=10.0 \mathrm{~Hz}, 2 \times \mathrm{CF}_{3}\right)$; MALDI TOF MS: $\mathrm{m} / \mathrm{z}$ calculated for $\left(\mathrm{C}_{47} \mathrm{H}_{24} \mathrm{BF}_{33} \mathrm{~N}_{2}\right)^{+}:$1254.2, found: 1254.1, $\mathrm{m} / z$ calculated for $\left(\mathrm{C}_{47} \mathrm{H}_{24} \mathrm{BF}_{32} \mathrm{~N}_{2}\right)^{+}:$1235.2, found: 1235.1; IR (ATR): $\tilde{v}\left(\mathrm{~cm}^{-1}\right)=2929(\mathrm{vw}), 1657(\mathrm{w}), 1546$ (m), $1499(\mathrm{~m}), 1464$ (w), 1393 (w), 1365 (w), 1318 (w), 1237 (m), 1173 (m), 1140 (m), 1101 (m), 1066 (m), 987 (m), 864 (w), 814 (w), $793(\mathrm{w}), 776(\mathrm{w}), 741(\mathrm{w}), 718(\mathrm{w}), 692(\mathrm{~m}), 672(\mathrm{w}), 630(\mathrm{w}), 569(\mathrm{~m}), 511(\mathrm{w})$.

\section{4,4-Difluoro-1,3,5,7-tetramethyl-2,6-bis $((E)-1 H, 2 H$-perfluorooct-1-en-1-yl)-8-} (perfluorophenyl)-4-bora-3a,4a-diaza-s-indacene (5) and 4,4-difluoro-1,3,5,7-tetramethyl2-((E)-1H,2H-perfluorooct-1-en-1-yl)-8-(perfluorophenyl)-4-bora-3a,4a-diaza-s-indacene (6):

BODIPY dye $1(100 \mathrm{mg}, 241 \mu \mathrm{mol}), 1 H, 1 H, 2 H$-perfluoro-1-octene (549 $\mu \mathrm{L}, 2.41 \mathrm{mmol})$, $\mathrm{Pd}(\mathrm{OAc})_{2}(10.8 \mathrm{mg}, 48.2 \mu \mathrm{mol})$ and tert-butyl peroxybenzoate $(93.6 \mathrm{mg}, 482 \mu \mathrm{mol})$ were

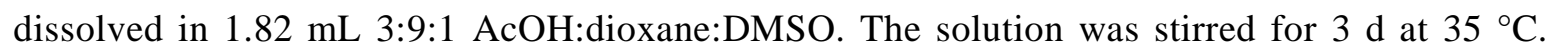
Afterwards a second portion of $\mathrm{Pd}(\mathrm{OAc})_{2}(5.4 \mathrm{mg}, 24.2 \mu \mathrm{mol})$ was added and the stirring was continued for additional $2 \mathrm{~d}$ at $35^{\circ} \mathrm{C}$. After cooling to ambient temperature, $10 \mathrm{~mL}$ water and 20 $\mathrm{mL}$ ethyl acetate were added. The organic layer was separated and evaporated under reduced pressure. The crude product was purified by using column chromatography (eluent 
cyclohexane/EtOAc 50:1) to give 5 as a red solid $(98.5 \mathrm{mg}, 37 \%)$ and $\mathbf{6}$ as a red solid (97.8 mg, $53 \%)$.

5: M.p. $175{ }^{\circ} \mathrm{C} ;{ }^{1} \mathrm{H}$ NMR $\left(400 \mathrm{MHz}, \mathrm{CDCl}_{3}\right): \delta=1.70(\mathrm{~s}, 6 \mathrm{H}), 2.70(\mathrm{~s}, 6 \mathrm{H}), 5.85(\mathrm{dt}, J(\mathrm{H}, \mathrm{H})=$ $16.4 \mathrm{~Hz}, J(\mathrm{H}, \mathrm{F})=11.7 \mathrm{~Hz}, 2 \mathrm{H}), 6.99(\mathrm{dt}, J(\mathrm{H}, \mathrm{H})=16.4 \mathrm{~Hz}, J(\mathrm{H}, \mathrm{F})=2.2 \mathrm{~Hz}, 2 \mathrm{H}) ;{ }^{13} \mathrm{C} \mathrm{NMR}(100$ $\left.\mathrm{MHz}, \mathrm{CDCl}_{3}\right): \delta=11.9\left(\mathrm{CH}_{3}\right), 14.1\left(\mathrm{CH}_{3}\right), 117.5(\mathrm{t}, J(\mathrm{C}, \mathrm{F})=23.2 \mathrm{~Hz}, \mathrm{CH}), 124.7(\mathrm{C}), 126.6(\mathrm{C})$, $129.7(\mathrm{t}, J(\mathrm{C}, \mathrm{F})=10.1 \mathrm{~Hz}, \mathrm{CH}), 131.2(\mathrm{C}), 139.7(\mathrm{C}), 158.3(\mathrm{C}) ;{ }^{19} \mathrm{~F}$ NMR $\left(376.5 \mathrm{MHz}, \mathrm{CDCl}_{3}\right)$ : $\delta=-158.3\left(\mathrm{~m}, 2 \times \mathrm{CF}_{\mathrm{ar}}\right),-148.6\left(\mathrm{t}, J(\mathrm{~F}, \mathrm{~F})=21 \mathrm{~Hz}, \mathrm{CF}_{\mathrm{ar}}\right),-144.0\left(\mathrm{q}, J(\mathrm{~F}, \mathrm{~B})=31.6 \mathrm{~Hz}, \mathrm{BF}_{2}\right),-$ $138.9\left(\mathrm{~m}, 2 \times \mathrm{CF}_{\mathrm{ar}}\right),-126.1\left(\mathrm{~m}, 2 \times \mathrm{CF}_{2}\right),-123.1\left(\mathrm{~m}, 2 \times \mathrm{CF}_{2}\right),-122.8\left(\mathrm{~m}, 2 \times \mathrm{CF}_{2}\right),-121.5(\mathrm{~m}$, $\left.2 \times \mathrm{CF}_{2}\right),-111.5\left(\mathrm{t}, J(\mathrm{~F}, \mathrm{~F})=11 \mathrm{~Hz}, 2 \times \mathrm{CF}_{2}\right),-80.8\left(\mathrm{t}, J(\mathrm{~F}, \mathrm{~F})=10.0 \mathrm{~Hz}, 2 \times \mathrm{CF}_{3}\right) ;$ MALDI TOF MS: $m / z$ calculated for $\left(\mathrm{C}_{35} \mathrm{H}_{16} \mathrm{BF}_{33} \mathrm{~N}_{2}\right)^{+}:$1102.1, found: 1102.0 , calculated for $\left(\mathrm{C}_{35} \mathrm{H}_{16} \mathrm{BF}_{32} \mathrm{~N}_{2}\right)^{+}$: 1083.1, found: 1083.0.

6: M.p. $149{ }^{\circ} \mathrm{C} ;{ }^{1} \mathrm{H}$ NMR $\left(400 \mathrm{MHz}, \mathrm{CDCl}_{3}\right): \delta=1.64(\mathrm{~s}, 3 \mathrm{H}), 1.68(\mathrm{~s}, 3 \mathrm{H}), 2.61(\mathrm{~s}, 3 \mathrm{H}), 2.66(\mathrm{~s}$, $3 \mathrm{H}), 5.80(\mathrm{dt}, J(\mathrm{H}, \mathrm{H})=16.4 \mathrm{~Hz}, J(\mathrm{H}, \mathrm{F})=11.9 \mathrm{~Hz}, 1 \mathrm{H}), 6.16(\mathrm{~s}, 1 \mathrm{H}), 6.99(\mathrm{dt}, J(\mathrm{H}, \mathrm{H})=16.4 \mathrm{~Hz}$, $J(\mathrm{H}, \mathrm{F})=2.2 \mathrm{~Hz}, 1 \mathrm{H}) ;{ }^{13} \mathrm{C} \mathrm{NMR}\left(100 \mathrm{MHz}, \mathrm{CDCl}_{3}\right): \delta=11.7\left(\mathrm{CH}_{3}\right), 13.9\left(\mathrm{CH}_{3}\right), 15.1\left(\mathrm{CH}_{3}\right)$, $116.0(\mathrm{t}, J(\mathrm{C}, \mathrm{F})=23.1 \mathrm{~Hz}, \mathrm{CH}), 123.6(\mathrm{C}), 123.9(\mathrm{C}), 125.0(\mathrm{C}), 129.8(\mathrm{C}), 130.2(\mathrm{t}, J(\mathrm{C}, \mathrm{F})=$ $10.1 \mathrm{~Hz}, \mathrm{CH}), 132.6$ (C), 137.7 (C), 143.7 (C), 155.1 (C), 161.3 (C); ${ }^{19} \mathrm{~F}$ NMR (376.5 MHz, $\left.\mathrm{CDCl}_{3}\right): \delta=-158.8\left(\mathrm{td}, J(\mathrm{~F}, \mathrm{~F})=21.0 \mathrm{~Hz}, J(\mathrm{~F}, \mathrm{~F})=6.8 \mathrm{~Hz}, 2 \times \mathrm{CF}_{\mathrm{ar}}\right),-149.5(\mathrm{t}, J(\mathrm{~F}, \mathrm{~F})=20.8 \mathrm{~Hz}$, $\left.\mathrm{CF}_{\mathrm{ar}}\right),-145.0\left(\mathrm{q}, J(\mathrm{~F}, \mathrm{~B})=31.9 \mathrm{~Hz}, \mathrm{BF}_{2}\right),-139.1\left(\mathrm{dd}, J(\mathrm{~F}, \mathrm{~F})=21.2 \mathrm{~Hz}, J(\mathrm{~F}, \mathrm{~F})=7.0 \mathrm{~Hz}, 2 \times \mathrm{CF}_{\mathrm{ar}}\right)$, $-126.1 \quad\left(\mathrm{~m}, \quad \mathrm{CF}_{2}\right), \quad-123.1 \quad\left(\mathrm{~m}, \quad \mathrm{CF}_{2}\right), \quad-122.8 \quad\left(\mathrm{~m}, \quad \mathrm{CF}_{2}\right), \quad-121.5 \quad\left(\mathrm{~m}, \quad \mathrm{CF}_{2}\right)$, $-111.2\left(\mathrm{t}, J(\mathrm{~F}, \mathrm{~F})=13 \mathrm{~Hz}, \mathrm{CF}_{2}\right),-80.7\left(\mathrm{t}, J(\mathrm{~F}, \mathrm{~F})=10.0 \mathrm{~Hz}, \mathrm{CF}_{3}\right)$; FAB MS: $m / z(\%): 758(100)$ $\left[\mathrm{M}^{+}\right], 739(51) \quad\left[(\mathrm{M}-\mathrm{F})^{+}\right], 489$ (22) $\quad\left[\left(\mathrm{M}-\mathrm{C}_{5} \mathrm{~F}_{11}\right)^{+}\right]$; HR FAB MS: $m / z$ calculated for $\left[\mathrm{C}_{27} \mathrm{H}_{15} \mathrm{BF}_{20} \mathrm{~N}_{2}\right]^{+}:$758.1009, found: 758.1011.

\section{2,6-Bis((4-bromophenyl)ethynyl)-4,4-difluoro-1,3,5,7-tetramethyl-8-(perfluorophenyl)-4- bora-3a,4a-diaza-s-indacene (7):}

BODIPY dye 2 (41.8 mg, $62.8 \mu \mathrm{mol})$, 1-bromo-4-ethynylbenzene (45.4 mg, $251 \mu \mathrm{mol})$, $\mathrm{Pd}\left(\mathrm{PPh}_{3}\right)_{2} \mathrm{Cl}_{2}(3.5 \mathrm{mg}, 4.99 \mu \mathrm{mol})$ and $\mathrm{CuI}(1.3 \mathrm{mg}, 6.91 \mu \mathrm{mol})$ were added to a vial. The vial was capped with a rubber septum and then purged with argon. Afterwards $150 \mu$ L DIPEA and 1

$\mathrm{mL}$ dry THF were added via a syringe and the mixture was stirred for $1.5 \mathrm{~d}$ at room temperature. Then, the mixture was partitioned between $25 \mathrm{~mL}$ dichloromethane and $10 \mathrm{~mL}$ water. The organic 
layer was separated and evaporated under reduced pressure. The crude product was purified by using column chromatography (eluent cyclohexane $/ \mathrm{CH}_{2} \mathrm{Cl}_{2}$ 8:1 $\rightarrow 4: 1$ ) to give a deep blue solid (43.9 mg, 91\%).

M.p. $>250^{\circ} \mathrm{C} ;{ }^{1} \mathrm{H}$ NMR $\left(400 \mathrm{MHz}, \mathrm{CDCl}_{3}\right): \delta=1.75(\mathrm{~s}, 6 \mathrm{H}), 2.72(\mathrm{~s}, 6 \mathrm{H}), 7.33(\mathrm{~d}, J(\mathrm{H}, \mathrm{H})=$ $8.5 \mathrm{~Hz}, 4 \mathrm{H}), 7.48(\mathrm{~d}, J(\mathrm{H}, \mathrm{H})=8.5 \mathrm{~Hz}, 4 \mathrm{H}) ;{ }^{13} \mathrm{C} \mathrm{NMR}\left(100 \mathrm{MHz}, \mathrm{CDCl}_{3}\right): \delta=12.6\left(\mathrm{CH}_{3}\right), 14.0$ $\left(\mathrm{CH}_{3}\right), 81.9$ (C), $96.4(\mathrm{C}), 117.2$ (C), 121.9 (C), 122.7 (C), 123.6 (C), 130.9 (C), $131.7\left(\mathrm{CH}_{\mathrm{ar}}\right)$, $132.8\left(\mathrm{CH}_{\mathrm{ar}}\right), 142.1(\mathrm{C}), 160.7(\mathrm{C}) ;{ }^{19} \mathrm{~F}$ NMR $\left(376.5 \mathrm{MHz}, \mathrm{CDCl}_{3}\right): \delta=-158.7(\mathrm{td}, J(\mathrm{~F}, \mathrm{~F})=21 \mathrm{~Hz}$, $\left.J(\mathrm{~F}, \mathrm{~F})=6 \mathrm{~Hz}, 2 \times \mathrm{CF}_{\mathrm{ar}}\right),-149.2\left(\mathrm{t}, J(\mathrm{~F}, \mathrm{~F})=21 \mathrm{~Hz}, \mathrm{CF}_{\mathrm{ar}}\right),-146.2\left(\mathrm{q}, J(\mathrm{~F}, \mathrm{~B})=31 \mathrm{~Hz}, \mathrm{BF}_{2}\right),-$ $138.9\left(\mathrm{dd}, J(\mathrm{~F}, \mathrm{~F})=21 \mathrm{~Hz}, J(\mathrm{~F}, \mathrm{~F})=6 \mathrm{~Hz}, 2 \times \mathrm{CF}_{\mathrm{ar}}\right) ; \mathrm{FAB} \mathrm{MS}: m / z(\%): 774 / 772 / 770(37 / 100 / 38)$ $\left[\mathrm{M}^{+}\right], 755 / 753 / 751(9 / 19 / 11)\left[(\mathrm{M}-\mathrm{F})^{+}\right]$; HR FAB MS: $m / z$ calculated for $\left(\mathrm{C}_{35} \mathrm{H}_{20} \mathrm{BBr}_{2} \mathrm{~F}_{7} \mathrm{~N}_{2}\right)^{+}$: 771.9955, found: 771.9956; IR (ATR): $\tilde{v}\left(\mathrm{~cm}^{-1}\right)=2926$ (vw), $2212(\mathrm{vw}), 1654(\mathrm{vw}), 1539(\mathrm{~m})$, 1500 (m), 1485 (m), 1433 (w), 1392 (w), 1317 (m), 1263 (m), 1176 (m), 1072 (m), 1046 (w), 993 (m), 863 (w), $820(\mathrm{~m}), 772(\mathrm{w}), 730(\mathrm{w}), 715(\mathrm{w}), 629(\mathrm{w}), 581(\mathrm{~m}), 472(\mathrm{vw}), 445$ (vw), 428 (w).

\section{4,4-Difluoro-1,3,5,7-tetramethyl-2,6-bis((4'-((E)-1H,2H-perfluorooct-1-en-1-yl)-[1,1'- biphenyl]-4-yl)ethynyl)-8-(perfluorophenyl)-4-bora-3a,4a-diaza-s-indacene (8):}

BODIPY dye 7 (30.3 mg, $39.2 \mu \mathrm{mol})$, fluorous boronic acid MIDA ester 3 (68.1 mg, $118 \mu \mathrm{mol}$ ) and $\mathrm{Pd}\left(\mathrm{PPh}_{3}\right)_{4}(4.5 \mathrm{mg}, 3.92 \mu \mathrm{mol})$ were added to a vial. The vial was capped with a rubber septum and then purged with argon. Afterwards $1.2 \mathrm{~mL}$ toluene (degassed by bubbling with argon for $30 \mathrm{~min}$ ) was added via a syringe and stirred for $10 \mathrm{~min}$ at room temperature. Then, $290 \mu \mathrm{L}$ aq. $\mathrm{NaOH}$ (3.0 M, degassed by bubbling with argon for $30 \mathrm{~min}$ ) was added and the mixture was stirred for $2.5 \mathrm{~h}$ at $110{ }^{\circ} \mathrm{C}$. After cooling to ambient temperature, the mixture was partitioned between $10 \mathrm{~mL}$ dichloromethane and $5 \mathrm{~mL}$ water. The organic layer was separated and evaporated under reduced pressure. The crude product was purified by using column chromatography (eluent cyclohexane/ $\mathrm{CH}_{2} \mathrm{Cl}_{2} 5: 1 \rightarrow 2: 1$ ) to give a deep blue solid (27.5 $\mathrm{mg}$, $48 \%)$.

M.p. $>250{ }^{\circ} \mathrm{C} ;{ }^{1} \mathrm{H}$ NMR $\left(400 \mathrm{MHz}, \mathrm{CDCl}_{3}\right): \delta=1.79(\mathrm{~s}, 6 \mathrm{H}), 2.76(\mathrm{~s}, 6 \mathrm{H}), 6.25(\mathrm{dt}, J(\mathrm{H}, \mathrm{H})=$ $16.0 \mathrm{~Hz}, J(\mathrm{H}, \mathrm{F})=12.5 \mathrm{~Hz}, 2 \mathrm{H}), 7.21(\mathrm{~d}, J(\mathrm{H}, \mathrm{H})=16.0 \mathrm{~Hz}, 2 \mathrm{H}), 7.55-7.66(\mathrm{~m}, 16 \mathrm{H}) ;{ }^{13} \mathrm{C} \mathrm{NMR}$ $\left(100 \mathrm{MHz}, \mathrm{CDCl}_{3}\right): \delta=12.6\left(\mathrm{CH}_{3}\right), 14.0\left(\mathrm{CH}_{3}\right), 82.0(\mathrm{C}), 97.2(\mathrm{C}), 114.5(\mathrm{t}, J(\mathrm{C}, \mathrm{F})=23 \mathrm{~Hz}$, $\mathrm{CH}), 117.4(\mathrm{C}), 122.6(\mathrm{C}), 123.5(\mathrm{C}), 127.0(\mathrm{CH}), 127.5(\mathrm{CH}), 128.2(\mathrm{CH}), 130.9(\mathrm{C}), 132.0$ $(\mathrm{CH}), 132.9(\mathrm{C}), 139.1(\mathrm{t}, J(\mathrm{C}, \mathrm{F})=10 \mathrm{~Hz}, \mathrm{CH}), 140.0(\mathrm{C}), 142.0(\mathrm{C}), 160.4(\mathrm{C}) ;{ }^{19} \mathrm{~F}$ NMR $(376.5$ 
$\left.\mathrm{MHz}, \mathrm{CDCl}_{3}\right): \delta=-158.8\left(\mathrm{~m}, 2 \times \mathrm{CF}_{\mathrm{ar}}\right),-149.3\left(\mathrm{t}, J(\mathrm{~F}, \mathrm{~F})=21 \mathrm{~Hz}, \mathrm{CF}_{\mathrm{ar}}\right),-146.2(\mathrm{q}, J(\mathrm{~F}, \mathrm{~B})=31$ $\left.\mathrm{Hz}, \mathrm{BF}_{2}\right),-138.9\left(\mathrm{~m}, 2 \times \mathrm{CF}_{\mathrm{ar}}\right),-126.1\left(\mathrm{~m}, 2 \times \mathrm{CF}_{2}\right),-123.1\left(\mathrm{~m}, 2 \times \mathrm{CF}_{2}\right),-122.8\left(\mathrm{~m}, 2 \times \mathrm{CF}_{2}\right)$, $-121.5\left(\mathrm{~m}, 2 \times \mathrm{CF}_{2}\right),-111.4\left(\mathrm{t}, J(\mathrm{~F}, \mathrm{~F})=12 \mathrm{~Hz}, 2 \times \mathrm{CF}_{2}\right),-80.7\left(\mathrm{t}, J(\mathrm{~F}, \mathrm{~F})=10.0 \mathrm{~Hz}, 2 \times \mathrm{CF}_{3}\right)$; MALDI TOF MS: $\mathrm{m} / z$ calculated for $\left(\mathrm{C}_{63} \mathrm{H}_{32} \mathrm{BF}_{33} \mathrm{~N}_{2}\right)^{+}: 1454.2$, found: $1454.2, \mathrm{~m} / z$ calculated for $\left(\mathrm{C}_{63} \mathrm{H}_{32} \mathrm{BF}_{32} \mathrm{~N}_{2}\right)^{+}:$1435.2, found: 1435.1 .

Scheme S1. Synthesis of alkyne $\mathbf{9}^{a}$

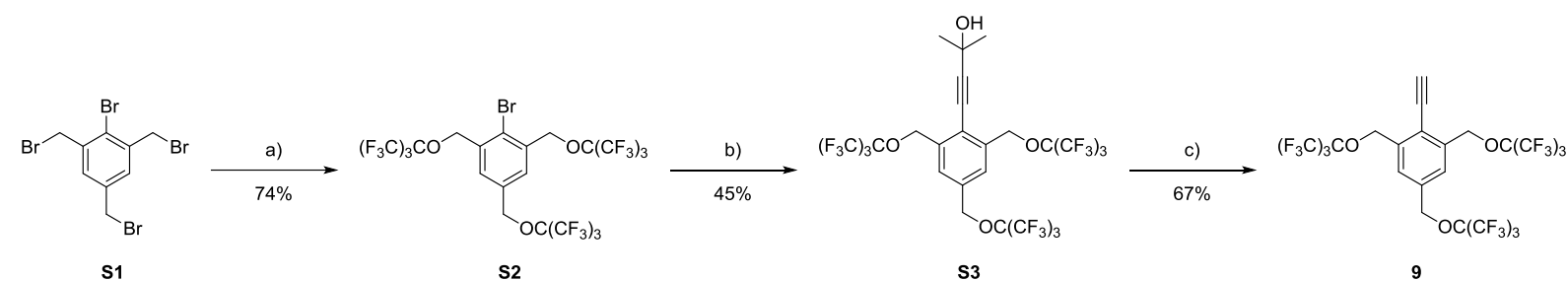

${ }^{a}$ Conditions: (a) sodium perfluoro-tert-butanolate, DMF, rt, overnight; (b) 2-methyl-3-butyn-2ol, $\mathrm{Pd}\left(\mathrm{PPh}_{3}\right)_{2} \mathrm{Cl}_{2}, \mathrm{CuI}, \mathrm{NEt}_{3}, 65^{\circ} \mathrm{C}, 20 \mathrm{~h}$; (c) $\mathrm{NBu}_{4} \mathrm{OH}, \mathrm{MeOH}$, toluene, $75^{\circ} \mathrm{C}, 2 \mathrm{~h}$.

\section{2-Bromo-1,3,5-tris(bromomethyl)benzene (S1):}

The preparation and properties of compound $\mathbf{S 1}$ have been reported in reference 3. Single crystals that were suitable for X-ray crystallography could be obtained by recrystallization from cyclohexane.

\section{2-Bromo-1,3,5-tris((perfluoro-tert-butoxy)methyl)benzene (S2):}

Compound $\mathbf{S 2}$ was prepared by using a procedure that was recently published. ${ }^{3}$ However, the procedure had to be modified in order to obtain the desired product. Thereby, the yield was improved as well (74\% vs. 52\%). In essence, sodium perfluoro-tert-butanolate ${ }^{4}$ (980 mg, $3.80 \mathrm{mmol}$ ) was added to a solution of 2-bromo-1,3,5-tris(bromomethyl)benzene (S1) (500 mg, $1.15 \mathrm{mmol})$ in dry DMF $(2 \mathrm{~mL})$. The mixture was stirred overnight at room temperature. Subsequently, water $(15 \mathrm{~mL})$ was added and the product was extracted with perfluorohexane (3 $\times 5 \mathrm{~mL})$. The fluorous phase was washed with brine $(10 \mathrm{~mL})$, water $(2 \times 5 \mathrm{~mL})$, and toluene 
( $5 \mathrm{~mL}$ ). The solvent was removed under reduced pressure, and $\mathbf{S 2}$ was obtained as a colorless oil (767 mg, 74\%). The analytical data were identical with the previously published data. ${ }^{3}$

\section{2-Methyl-4-(2,4,6-tris((perfluoro-tert-butoxy)methyl)phenyl)but-3-yn-2-ol (S3):}

2-Bromo-1,3,5-tris((perfluoro-tert-butoxy)methyl)benzene (S2) $(586 \mathrm{mg}, 650 \mu \mathrm{mol}), 2$-methyl3-butyn-2-ol $(83.4 \mu \mathrm{L}, 853 \mu \mathrm{mol}), \mathrm{Pd}\left(\mathrm{PPh}_{3}\right)_{2} \mathrm{Cl}_{2}(9.2 \mathrm{mg}, 13.1 \mu \mathrm{mol})$ and $\mathrm{CuI}(5.0 \mathrm{mg}, 26.2 \mu \mathrm{mol})$ were added to a vial. The vial was capped with a rubber septum and then purged with argon. Afterwards $3.3 \mathrm{~mL}$ dry $\mathrm{NEt}_{3}$ were added via a syringe and the mixture was stirred for $20 \mathrm{~h}$ at $65^{\circ} \mathrm{C}$. After cooling to ambient temperature, the solvent was evaporated under reduced pressure. The crude product was purified by using column chromatography (eluent cyclohexane/EtOAc $8: 1)$ to give a yellowish solid $(262 \mathrm{mg}, 45 \%)$.

M.p. $65{ }^{\circ} \mathrm{C} ;{ }^{1} \mathrm{H}$ NMR (400 MHz, $\left.\mathrm{CDCl}_{3}\right): \delta=1.62$ (s, 6H), 1.96 (bs, $\left.1 \mathrm{H}\right), 5.11(\mathrm{~s}, 2 \mathrm{H}), 5.22$ (s, 4H), 7.43 (s 2H); $\left.{ }^{13} \mathrm{C} \mathrm{NMR} \mathrm{(100} \mathrm{MHz,} \mathrm{CDCl}_{3}\right): \delta=31.1\left(2 \times \mathrm{CH}_{3}\right), 65.7(\mathrm{C}), 68.8(\mathrm{~d}, J(\mathrm{C}, \mathrm{F})=$ $\left.1.4 \mathrm{~Hz}, 2 \times \mathrm{CH}_{2}\right), 70.2\left(\mathrm{~d}, J(\mathrm{C}, \mathrm{F})=1.4 \mathrm{~Hz}, \mathrm{CH}_{2}\right), 74.9(\mathrm{C}), 80.0($ decet, $J(\mathrm{C}, \mathrm{F})=29.9 \mathrm{~Hz}, 3 \times \mathrm{C})$, $105.8(\mathrm{C}), 119.6(\mathrm{Car}), 120.4\left(\mathrm{q}, J(\mathrm{C}, \mathrm{F})=294.8 \mathrm{~Hz}, 9 \times \mathrm{CF}_{3}\right), 125.5\left(2 \times \mathrm{CH}_{\mathrm{ar}}\right), 136.1\left(\mathrm{C}_{\mathrm{ar}}\right), 137.6$ $\left(2 \times \mathrm{C}_{\mathrm{ar}}\right) ;{ }^{19} \mathrm{~F} \mathrm{NMR}\left(376.5 \mathrm{MHz}, \mathrm{CDCl}_{3}\right): \delta=-70.3\left(\mathrm{~s}, 3 \times \mathrm{CF}_{3}\right),-70.2\left(\mathrm{~s}, 6 \times \mathrm{CF}_{3}\right)$; EI MS: $\mathrm{m} / z$ (\%): 904 (71) $\left[\mathrm{M}^{+}\right], 889$ (39) $\left[(\mathrm{M}-\mathrm{CH} 3)^{+}\right], 885(26)\left[(\mathrm{M}-\mathrm{F})^{+}\right], 669(85)\left[\left(\mathrm{M}-\mathrm{C}_{4} \mathrm{~F}_{9} \mathrm{O}\right)^{+}\right], 655(96)$ $\left[\left(\mathrm{M}-\mathrm{C}_{5} \mathrm{HF}_{9} \mathrm{O}\right)^{+}\right], \quad 653$ (100) $\quad\left[\left(\mathrm{M}-\mathrm{C}_{4} \mathrm{~F}_{9} \mathrm{O}_{2}\right)^{+}\right], 611 \quad(43) \quad\left[\left(\mathrm{M}-\mathrm{C}_{7} \mathrm{H}_{6} \mathrm{~F}_{9} \mathrm{O}_{2}\right)^{+}\right], \quad 449$ (50) $\quad[(\mathrm{M}-$ $\left.\left.\mathrm{C}_{8} \mathrm{HF}_{18} \mathrm{O}\right)^{+}\right], 417(36)\left[\left(\mathrm{M}-\mathrm{C}_{8} \mathrm{HF}_{18} \mathrm{O}_{3}\right)^{+}\right]$; HR EI MS: $m / z$ calculated for $\left[\mathrm{C}_{26} \mathrm{H}_{15} \mathrm{~F}_{27} \mathrm{O}_{4}\right]^{+}: 904.0539$, found: 904.0540; IR (ATR): $\tilde{v}\left(\mathrm{~cm}^{-1}\right)=3308$ (vw), 2986 (vw), 1470 (vw), 1387 (vw), 1243 (s), 1146 (s), 1026 (m), 968 (s), 910 (w), 871 (w), 804 (vw), 772 (vw), 735 (m), 726 (s), 633 (vw), $571(\mathrm{vw}), 538(\mathrm{w}), 492(\mathrm{w}), 441(\mathrm{vw})$.

\section{2-Ethynyl-1,3,5-tris((perfluoro-tert-butoxy)methyl)benzene (9):}

2-Methyl-4-(2,4,6-tris((perfluoro-tert-butoxy)methyl)phenyl)but-3-yn-2-ol (S3) (218 mg, 241 $\mu \mathrm{mol})$ was dissolved in $11 \mathrm{~mL}$ toluene under argon atmosphere. To this solution $\mathrm{NBu}_{4} \mathrm{OH}(1 \mathrm{M}$ in $\mathrm{MeOH}, 24 \mu \mathrm{L}, 24 \mu \mathrm{mol}$ ) was added and the reaction mixture was stirred at $75{ }^{\circ} \mathrm{C}$ for $60 \mathrm{~min}$. Afterwards a second portion of $\mathrm{NBu}_{4} \mathrm{OH}(1 \mathrm{M}$ in $\mathrm{MeOH}, 10 \mu \mathrm{L}, 10 \mu \mathrm{mol})$ was added and the mixture was stirred for an additional $60 \mathrm{~min}$ at $75{ }^{\circ} \mathrm{C}$. After cooling to ambient temperature, the solvent was reduced to approximately $1 \mathrm{~mL}$. The product was extracted with $2 \times 3 \mathrm{~mL}$ perfluorohexane and washed with $1 \mathrm{~mL}$ water and $1 \mathrm{~mL}$ toluene afterwards. The solvent was 
removed under reduced pressure and the crude product was purified by using column chromatography (eluent cyclohexane) to give a white solid (137 mg, 67\%).

M.p. $47{ }^{\circ} \mathrm{C} ;{ }^{1} \mathrm{H}$ NMR $\left(400 \mathrm{MHz}, \mathrm{CDCl}_{3}\right): \delta=3.65$ (s, 1H), 5.12 (s, 2H), 5.26 (s, 4H), 7.46 (s, $2 \mathrm{H}) ;{ }^{13} \mathrm{C} \mathrm{NMR}\left(100 \mathrm{MHz}, \mathrm{CDCl}_{3}\right): \delta=68.7\left(2 \times \mathrm{CH}_{2}\right), 70.2\left(\mathrm{CH}_{2}\right), 76.1(\mathrm{CH}), 80.0(\operatorname{decet}, J(\mathrm{C}, \mathrm{F})$ $=29.6 \mathrm{~Hz}, 3 \times \mathrm{C}), 89.0(\mathrm{C}), 118.6\left(\mathrm{C}_{\mathrm{ar}}\right), 120.3\left(\mathrm{q}, J(\mathrm{C}, \mathrm{F})=293.1 \mathrm{~Hz}, 9 \times \mathrm{CF}_{3}\right), 125.3\left(2 \times \mathrm{CH}_{\mathrm{ar}}\right)$, 136.6 $\left(\mathrm{C}_{\mathrm{ar}}\right), 138.4\left(2 \times \mathrm{C}_{\mathrm{ar}}\right) ;{ }^{19} \mathrm{~F} \mathrm{NMR}\left(376.5 \mathrm{MHz}, \mathrm{CDCl}_{3}\right): \delta=-70.3\left(\mathrm{~s}, 3 \times \mathrm{CF}_{3}\right)$, $-70.2\left(\mathrm{~s}, 6 \times \mathrm{CF}_{3}\right)$; EI MS: $m / z(\%): 846(90)\left[\mathrm{M}^{+}\right], 827(21)\left[(\mathrm{M}-\mathrm{F})^{+}\right], 627(95)\left[\left(\mathrm{M}-\mathrm{C}_{4} \mathrm{~F}_{9}\right)^{+}\right], 611$ (100) $\left[\left(\mathrm{M}-\mathrm{C}_{4} \mathrm{~F}_{9} \mathrm{O}\right)^{+}\right], \quad 597(58)\left[\left(\mathrm{M}-\mathrm{C}_{5} \mathrm{H}_{2} \mathrm{~F}_{9} \mathrm{O}\right)^{+}\right], 407$ (11) $\left[\left(\mathrm{M}_{-} \mathrm{C}_{8} \mathrm{HF}_{18}\right)^{+}\right], 391$ (25) $[(\mathrm{M}-$ $\left.\left.\mathrm{C}_{8} \mathrm{HF}_{18} \mathrm{O}\right)^{+}\right], 188(23)\left[\left(\mathrm{M}-\mathrm{C}_{12} \mathrm{HF}_{27}\right)^{+}\right]$; HR EI MS: $m / z$ calculated for $\left[\mathrm{C}_{23} \mathrm{H}_{9} \mathrm{~F}_{27} \mathrm{O}_{3}\right]^{+}:$846.0121, found: 846.0119; IR (ATR): $\tilde{v}\left(\mathrm{~cm}^{-1}\right)=3313$ (vw), 1469 (vw), 1391 (vw), 1240 (s), 1136 (s), $1023(\mathrm{~m}), 1007$ (m), 968 (s), $884(\mathrm{w}), 871(\mathrm{w}), 776(\mathrm{w}), 725$ (s), $668(\mathrm{~m}), 635(\mathrm{~m}), 571(\mathrm{vw}), 538$ (m), $500(\mathrm{w}), 459(\mathrm{w}), 436(\mathrm{vw})$.

\section{4,4-Difluoro-1,3,5,7-tetramethyl-8-(perfluorophenyl)-2,6-bis((2,4,6-tris((perfluoro-tert- butoxy)methyl)phenyl)ethynyl)-4-bora-3a,4a-diaza-s-indacene (10):}

BODIPY dye $\quad 2 \quad(35.8 \quad \mathrm{mg}, \quad 53.7 \quad \mu \mathrm{mol}), \quad$ 2-ethynyl-1,3,5-tris((perfluoro-tertbutoxy)methyl)benzene (9) (100 mg, $118 \mu \mathrm{mol}), \mathrm{Pd}\left(\mathrm{PPh}_{3}\right)_{2} \mathrm{Cl}_{2}(4.1 \mathrm{mg}, 5.91 \mu \mathrm{mol})$ and $\mathrm{CuI}(1.1$ $\mathrm{mg}, 5.91 \mu \mathrm{mol})$ were added to a vial. The vial was capped with a rubber septum and then purged with argon. Afterwards $150 \mu \mathrm{L}$ DIPEA and $1 \mathrm{~mL}$ dry THF were added via a syringe and the mixture was stirred for $2.5 \mathrm{~d}$ at room temperature. Then, the mixture was partitioned between 25 $\mathrm{mL} \mathrm{CH}_{2} \mathrm{Cl}_{2}$ and $10 \mathrm{~mL}$ water. The organic layer was separated and filtered through a plug of silica gel (eluent EtOAc). The solvent was evaporated under reduced pressure. The crude product was purified by washing with $15 \mathrm{~mL}$ acetonitrile and $10 \mathrm{~mL}$ acetonitrile/acetone (1:1) to give a dark blue solid (96.7 mg, 86\%).

M.p. $>250{ }^{\circ} \mathrm{C} ;{ }^{1} \mathrm{H}$ NMR $\left(500 \mathrm{MHz}, \mathrm{d}_{8}\right.$-THF): $\delta=1.87(\mathrm{~s}, 6 \mathrm{H}), 2.73(\mathrm{~s}, 6 \mathrm{H}), 5.28(\mathrm{~s}, 4 \mathrm{H}), 5.45$ (s, $8 \mathrm{H}), 7.62(\mathrm{~s}, 4 \mathrm{H}) ;{ }^{13} \mathrm{C}$ NMR was not obtained due to poor signal-to-noise ratio; ${ }^{19} \mathrm{~F}$ NMR (376.5 $\left.\mathrm{MHz}, \mathrm{d}_{8}-\mathrm{THF}\right): \delta=-155.3\left(\mathrm{t}, J(\mathrm{~F}, \mathrm{~F})=21 \mathrm{~Hz}, 2 \times \mathrm{CF}_{\mathrm{ar}}\right),-145.4\left(\mathrm{t}, J(\mathrm{~F}, \mathrm{~F})=21 \mathrm{~Hz}, \mathrm{CF}_{\mathrm{ar}}\right),-140.8$ $\left(\mathrm{q}, J(\mathrm{~F}, \mathrm{~B})=31 \mathrm{~Hz}, \mathrm{BF}_{2}\right),-136.3\left(\mathrm{~d}, J(\mathrm{~F}, \mathrm{~F})=21 \mathrm{~Hz}, 2 \times \mathrm{CF}_{\mathrm{ar}}\right),-65.6\left(\mathrm{~s}, 6 \times \mathrm{CF}_{3}\right),-65.4(\mathrm{~s}, 12 \times$ $\left.\mathrm{CF}_{3}\right)$; MALDI TOF MS: $\mathrm{m} / z$ calculated for $\left(\mathrm{C}_{65} \mathrm{H}_{28} \mathrm{BF}_{61} \mathrm{~N}_{2} \mathrm{O}_{6}\right)^{+}: 2102.1$, found: 2102.1 , calculated for $\left(\mathrm{C}_{65} \mathrm{H}_{28} \mathrm{BF}_{60} \mathrm{~N}_{2} \mathrm{O}_{6}\right)^{+}:$2083.1, found: 2083.2; IR (ATR): $\tilde{v}\left(\mathrm{~cm}^{-1}\right)=1539(\mathrm{~m}), 1499(\mathrm{w}), 1442$ (w), 1393 (w), 1249 (s), 1185 (m), 1149 (s), 1100 (m), 992 (s), 969 (s), 886 (w), 773 (w), 725 (s), $682(\mathrm{w}), 640(\mathrm{vw}), 607(\mathrm{vw}), 578(\mathrm{~m}), 498(\mathrm{w})$. 


\section{Computational Studies}

All quantum chemical calculations reported in this work were carried out with the TURBOMOLE program package ${ }^{5}$ using standard orbital basis sets of type "def2"6 and (timedependent) density functional theory methods (DFT and TDDFT).

The structures of dyes $1\left(\mathrm{C}_{2 \mathrm{v}}\right.$ symmetry), 4, 5, $\mathbf{6}$ and $\mathbf{8}$ (all $\mathrm{C}_{2}$ symmetry except for $\left.\mathbf{6}\right)$ with $\mathrm{R}_{\mathrm{f}}=$ $\left(\mathrm{CF}_{2}\right)_{5} \mathrm{CF}_{3}$ and dyes $4^{*}, \mathbf{5}^{*}, \mathbf{6}^{*}$ and $\mathbf{8}^{*}$ (all $\mathrm{C}_{2}$ symmetry except for $\mathbf{6}^{*}$ ) with $\mathrm{R}_{\mathrm{f}}=\mathrm{CF}_{3}$ were optimized at the $\mathrm{B} 3 \mathrm{LYP}^{7} / \mathrm{TZVP}$ level of theory using fine quadrature grids $(\mathrm{m} 4)$ and tight convergence criteria (SCF energy: $10^{-8} E_{\mathrm{h}}$, energy gradient: $10^{-4} E_{\mathrm{h}} / \mathrm{a}_{0}$ and inclusion of derivatives of quadrature weights). All obtained structures were confirmed to be minima at the potential energy surface through analysis of force constants and vibrational frequencies. Orbital plots were generated using isosurface values of \pm 0.07 .

Vertical excitation energies for the above-mentioned dyes $1,4^{*}, 5^{*}, 6^{*}$ and $8^{*}\left(\mathrm{R}_{\mathrm{f}}=\mathrm{CF}_{3}\right)$ were calculated within the framework of TDDFT response theory using the B3LYP and M06-2X functionals with diffuse augmented basis sets $\left(\mathrm{SVPD}^{9}\right)$. The COSMO model ${ }^{10}$ was applied to study solvent effects on the excitation energies $\left(\varepsilon=8.93, n=1.424\right.$ for $\left.\mathrm{CH}_{2} \mathrm{Cl}_{2}\right)$.

In Fig. S1 the computed HOMO-LUMO energy differences $(\Delta \mathrm{E})$ and the TDDFT excitation energies are plotted against the experimental data (the dashed line represents perfect agreement). All methods overestimate the excitation energies, with M06-2X+COSMO showing a systematic shift of about 0.4 $\mathrm{eV}$ for all investigated dyes. The B3LYP values for dye $8 *$ are very close to the measured value. However, this is due to the fact that this excitation has significant charge transfer character which is not properly described (that is, underestimated) by the B3LYP functional and thus the agreement is due to fortuitous error cancellation. 


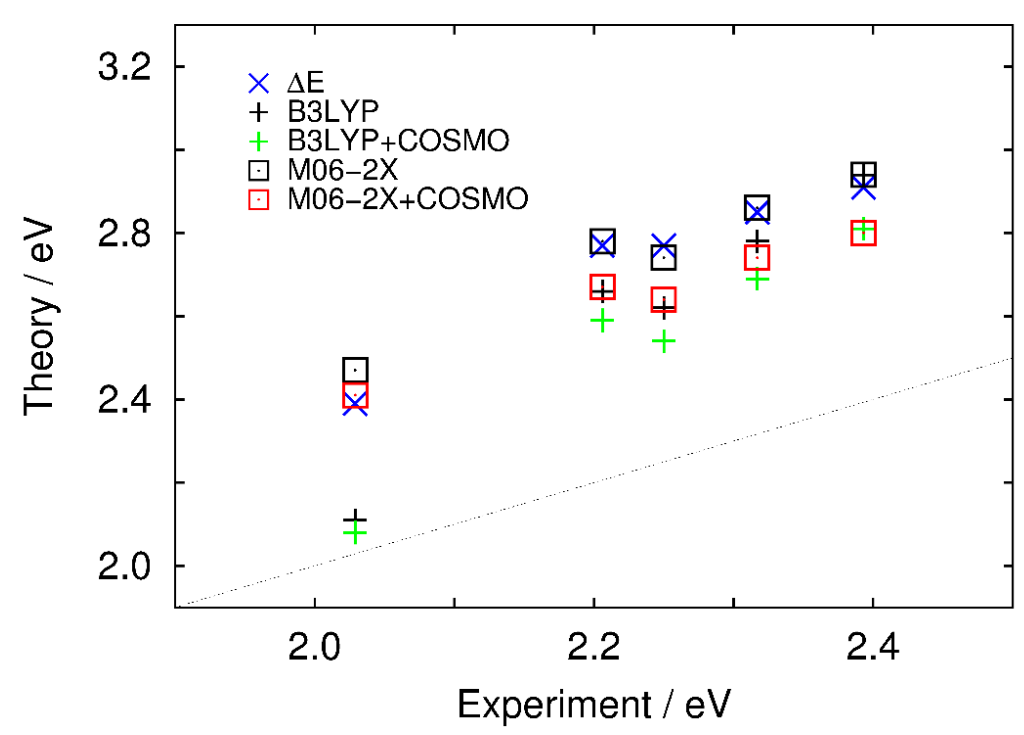

Figure S1. Comparison of computed and experimental excitation energies.

Fig. S2 shows several computed excitation energies in the range up to $200 \mathrm{~nm}$ and the corresponding oscillator strengths for dyes $1,4^{*}, 5^{*}, 6^{*}$ and $\mathbf{8}^{*}$. As already discussed, the lowest excitation energy corresponds to transitions with HOMO $\rightarrow$ LUMO character. For all dyes, we find transitions with HOMO-1 $\rightarrow$ LUMO and HOMO-2 $\rightarrow$ LUMO character which have less oscillator strengths. These transitions might contribute to the experimentally observed weak band at around 400-425 nm. Interestingly, we observe transitions with $\mathrm{HOMO} \rightarrow \mathrm{LUMO}+2$ (and partially $\mathrm{HOMO}-1 \rightarrow$ LUMO+1) character for all substituted dyes. These transitions show increasing oscillator strengths with extended $\pi$-electron system; for dye $\mathbf{8}^{*}$ this transition even has a larger oscillator strength than the $\mathrm{HOMO} \rightarrow$ LUMO transition. 

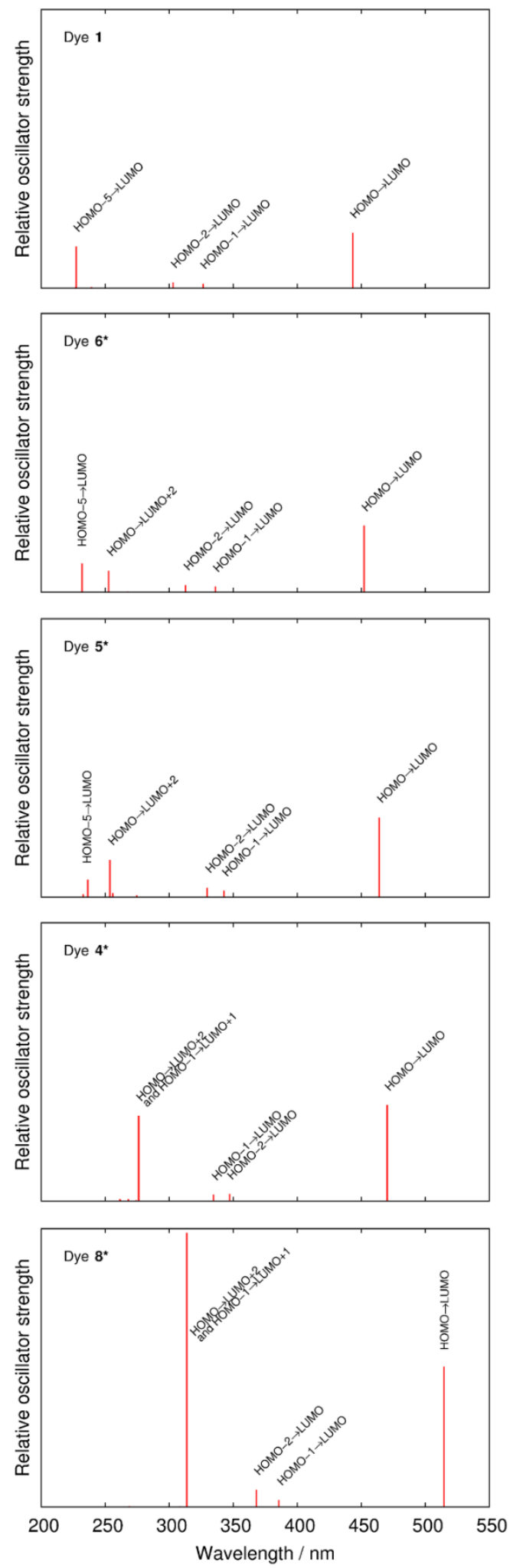

Figure S2. Computed absorption spectra and assignment of involved transitions based on M06$2 \mathrm{X}+\mathrm{COSMO}$ values. 


\section{Lifetime Fittings}

Fittings for lifetimes measured by either TCSPC (dyes 1, 4, 5, 7, 8, and 10) or transient absorption (dye 2) are shown below.
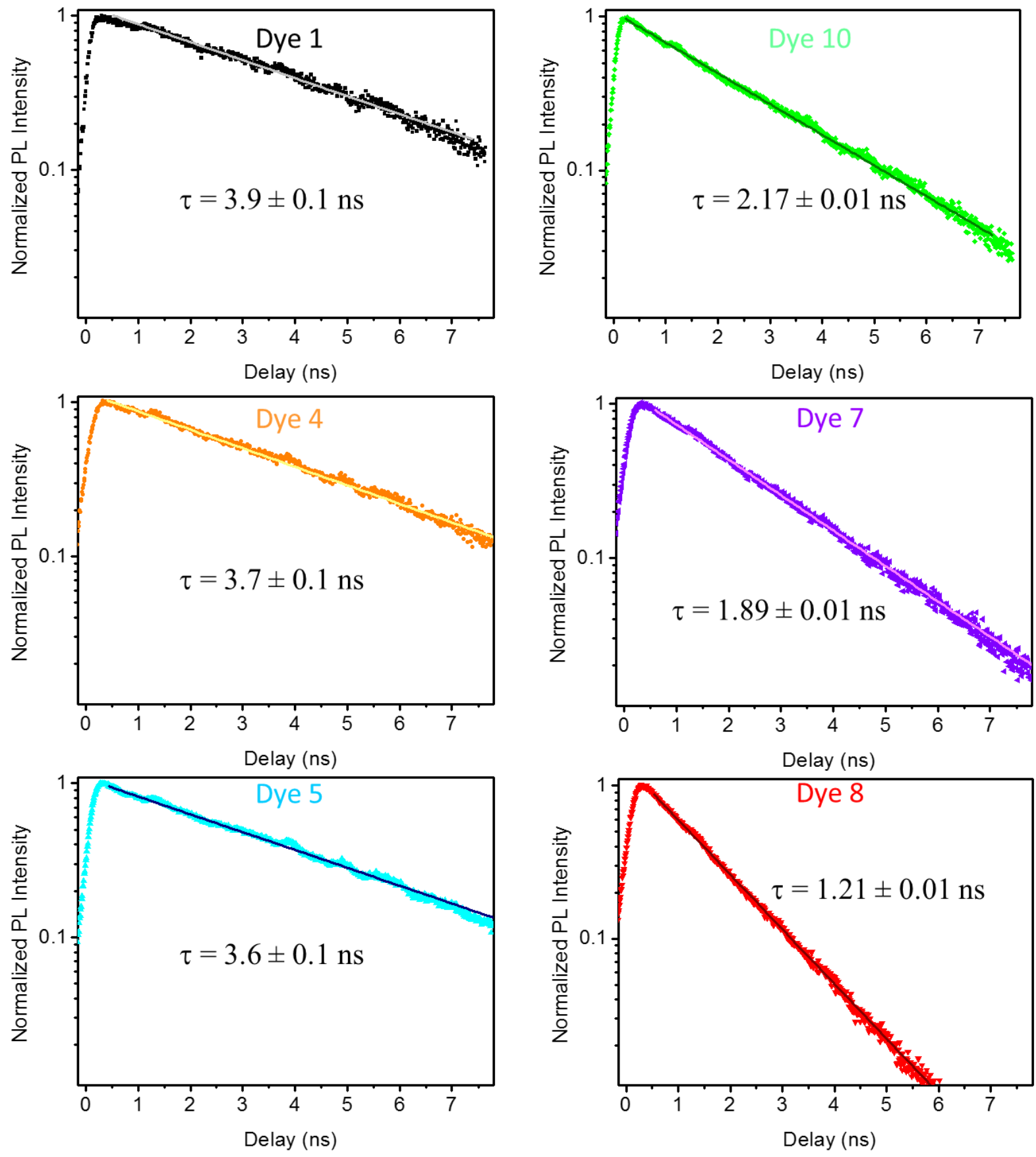

Figure S3. Fluorescence lifetimes measured by TCSPC for 6 of the investigated dyes. 


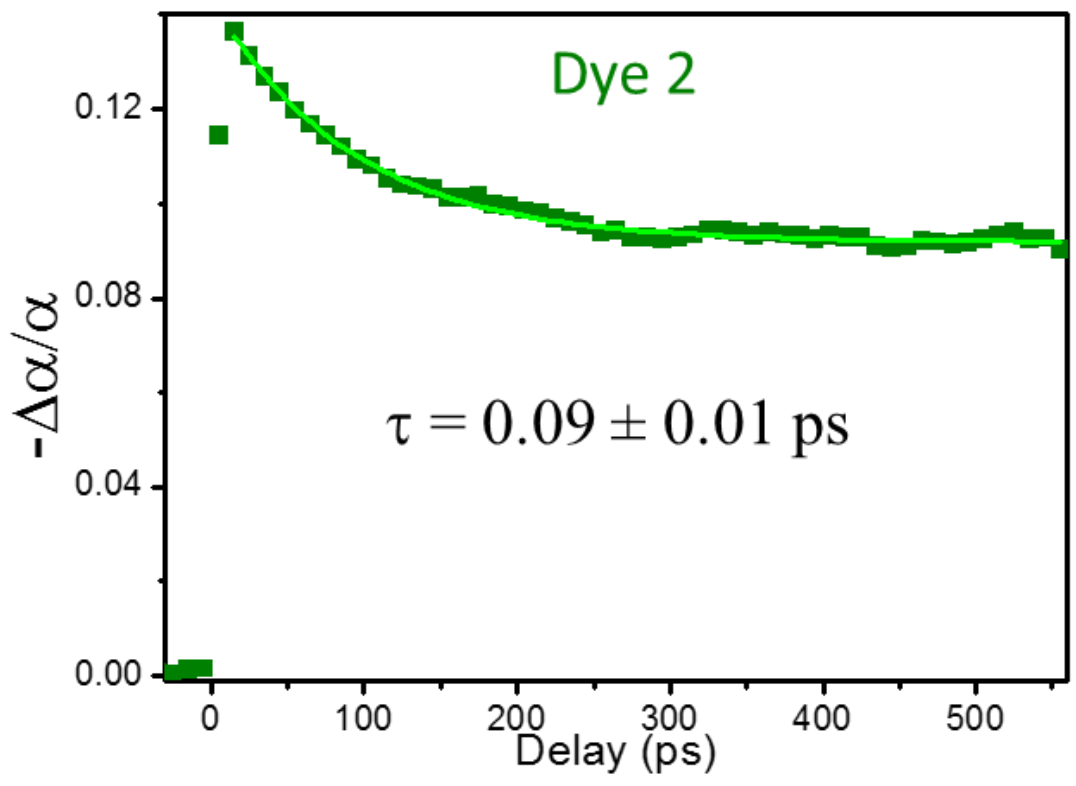

Figure S4. Intersystem crossing time measured by transient absorption for dye $\mathbf{2}$.

\section{Nonlinear Spectroscopy}

For the nonlinear spectroscopy studies we used an ultrafast laser system consisting of an amplified Ti:sapphire laser and an optical parametric amplifier (OPA) operating at $1 \mathrm{kHz}$ and delivering pulses of about 70-100 fs. The visible ESA spectra measurements were performed with a pump-probe setup where the probe is a white light continuum (WLC), covering the visible spectral range, generated in water. The WLC transmitted through the dye was measured with and without the presence of a pump beam. The pump excites the sample to the first excited state, and the ESA spectrum results from the difference between the absorption of the WLC with and without the presence of the pump. This method only gives the spectral shape of the ESA. To quantify the ESA cross section we performed open aperture Z-scans ${ }^{11}$ at a wavelength near the ESA peak and the data was fit considering a three-level model with only singlet states as described in detail in Ref. ${ }^{12}$. This approach is reasonable because the triplet states measured on dye $\mathbf{2}$ should be at lower energies. Examples of Z-scan curves and fittings are presented below. 


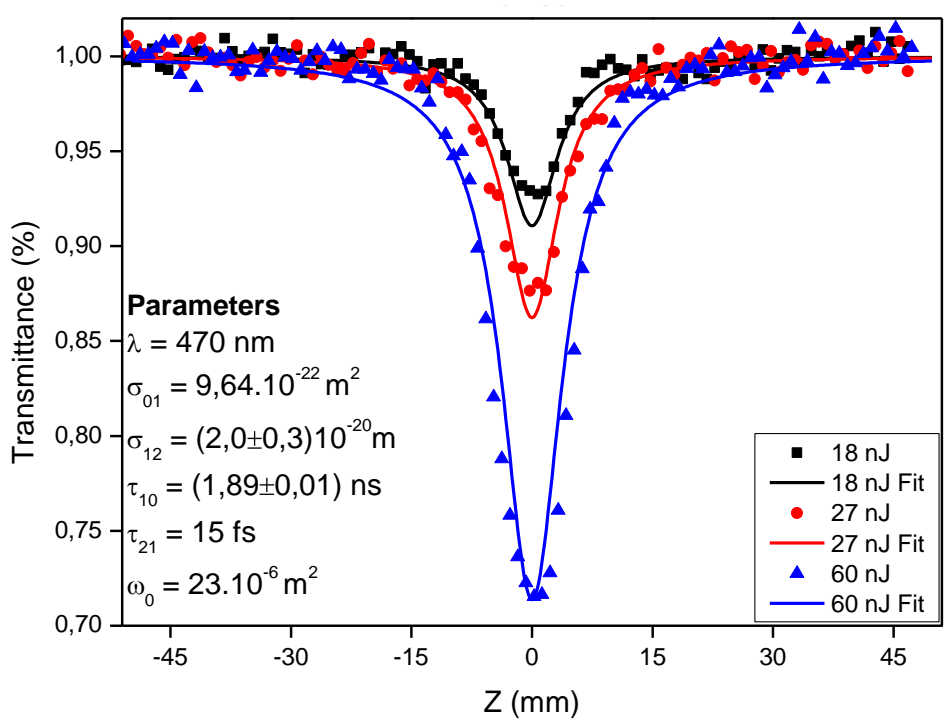

Figure S5. Example of an open aperture Z-scan for quantification of ESA. In this example, dye 4 is investigated with excitation at $470 \mathrm{~nm}$.

For all samples, Z-scans were performed for at least 3 pump energies, to verify the energy dependence of the signal and validate the model from Ref. ${ }^{12}$ which was used for fitting.

\section{Crystallographic Data}

\section{Crystal Structure Determinations}

The single-crystal X-ray diffraction study was carried out on a Bruker-Nonius KappaCCD at 123(2) K using Mo-K $\alpha$ radiation (2) and a Bruker Apex Duo at 120(2) K using Mo-K $\alpha$ radiation (S1) $(\lambda=0.71073 \AA)$. Direct Methods (SHELXS-97) $)^{13}$ were used for structure solution and refinement was carried out using SHELXL-98 ${ }^{13}$ (full-matrix least-squares on $F^{2}$ ). Hydrogen atoms were localized by difference electron density determination and refined using a riding model. Semi-empirical absorption corrections were applied. BODIPY dye $\mathbf{2}$ was obtained as a non-merohedral twin with two domains.

2: res crystals, $\mathrm{C}_{19} \mathrm{H}_{12} \mathrm{BF}_{7} \mathrm{I}_{2} \mathrm{~N}_{2}, M_{\mathrm{r}}=665.92$, crystal size $0.18 \times 0.06 \times 0.03 \mathrm{~mm}$, monoclinic, space group $P 2_{1} / \mathrm{n}$ (No. 14), $a=7.7898(3) \AA ⿻$, $b=22.2188(19) \AA, c=11.758(15) \AA, \beta=$ 97.490(7) ${ }^{\circ}, V=2017.7(3) \AA^{3}, Z=4, \rho=2.192 \mathrm{Mg} / \mathrm{m}^{-3}, \mu\left(\mathrm{mMo}-\mathrm{K}_{\alpha}\right)=3.190 \mathrm{~mm}^{-1}, F(000)=1256$, $2 \theta_{\max }=50^{\circ}, 3525$ reflections, of which 3525 were independent $\left(R_{\text {int }}=0.000\right.$, non-merohedral twin, use of HKLF 5 file), 285 parameters, $R_{1}=0.058$ (for $2810 \mathrm{I}>2 \sigma(\mathrm{I})$ ), $\mathrm{w} R_{2}=0.143$ (all data), 
$S=1.10$, largest diff. peak $/$ hole $=1.106 /-1.725$ e $\AA^{-3}$.

S1: colourless crystals, $\mathrm{C}_{9} \mathrm{H}_{8} \mathrm{Br}_{4}, M_{\mathrm{r}}=435.79$, crystal size $0.12 \times 0.06 \times 0.03 \mathrm{~mm}$, monoclinic, space group $P 2_{1} / \mathrm{c}$ (No. 14), $a=4.5681(3) \AA, b=17.2553(10) \AA, c=14.8063(11) \AA, \beta=$ 95.764(2) ${ }^{\circ}, V=1161.19(13) \AA^{3}, Z=4, \rho=2.493 \mathrm{Mg} / \mathrm{m}^{-3}, \mu\left(\mathrm{Mo}-\mathrm{K}_{\alpha}\right)=13.816 \mathrm{~mm}^{-1}, F(000)=$ $808,2 \theta_{\max }=55^{\circ}, 5132$ reflections, of which 2607 were independent $\left(R_{\mathrm{int}}=0.022\right), 118$ parameters, $R_{1}=0.027$ (for $2120 \mathrm{I}>2 \sigma(\mathrm{I})$ ), $\mathrm{w} R_{2}=0.062$ (all data), $S=1.04$, largest diff. peak / hole $=0.690 /-0.722 \mathrm{e}^{-3}$.

CCDC 1442275 (2), and 1442277 (S1) contain the supplementary crystallographic data for this paper. These data can be obtained free of charge from The Cambridge Crystallographic Data Centre via www.ccdc.cam.ac.uk/data_request/cif.

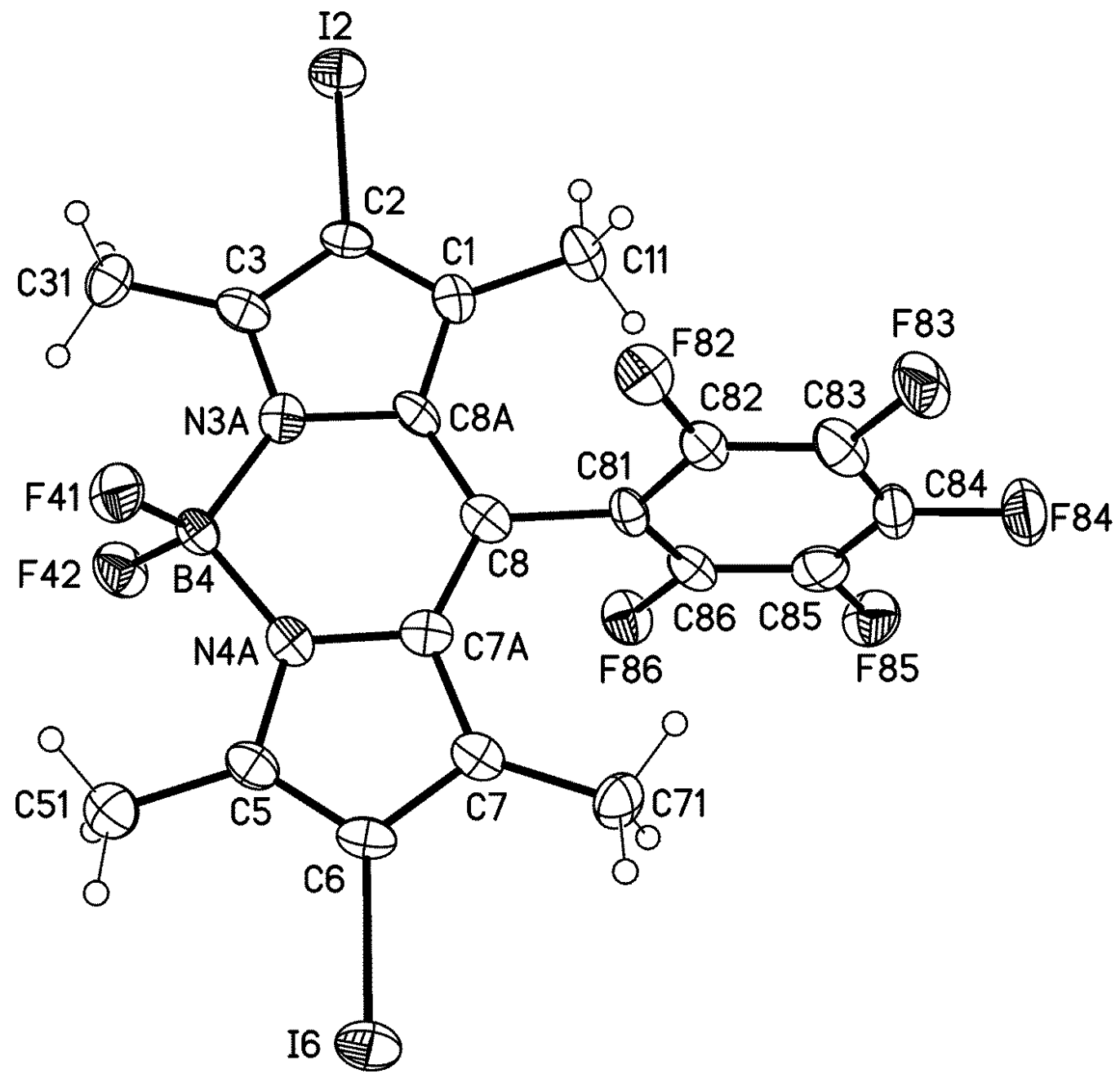

Figure S6. Molecular structure of 2 (displacement parameters are drawn at $50 \%$ probability level). 


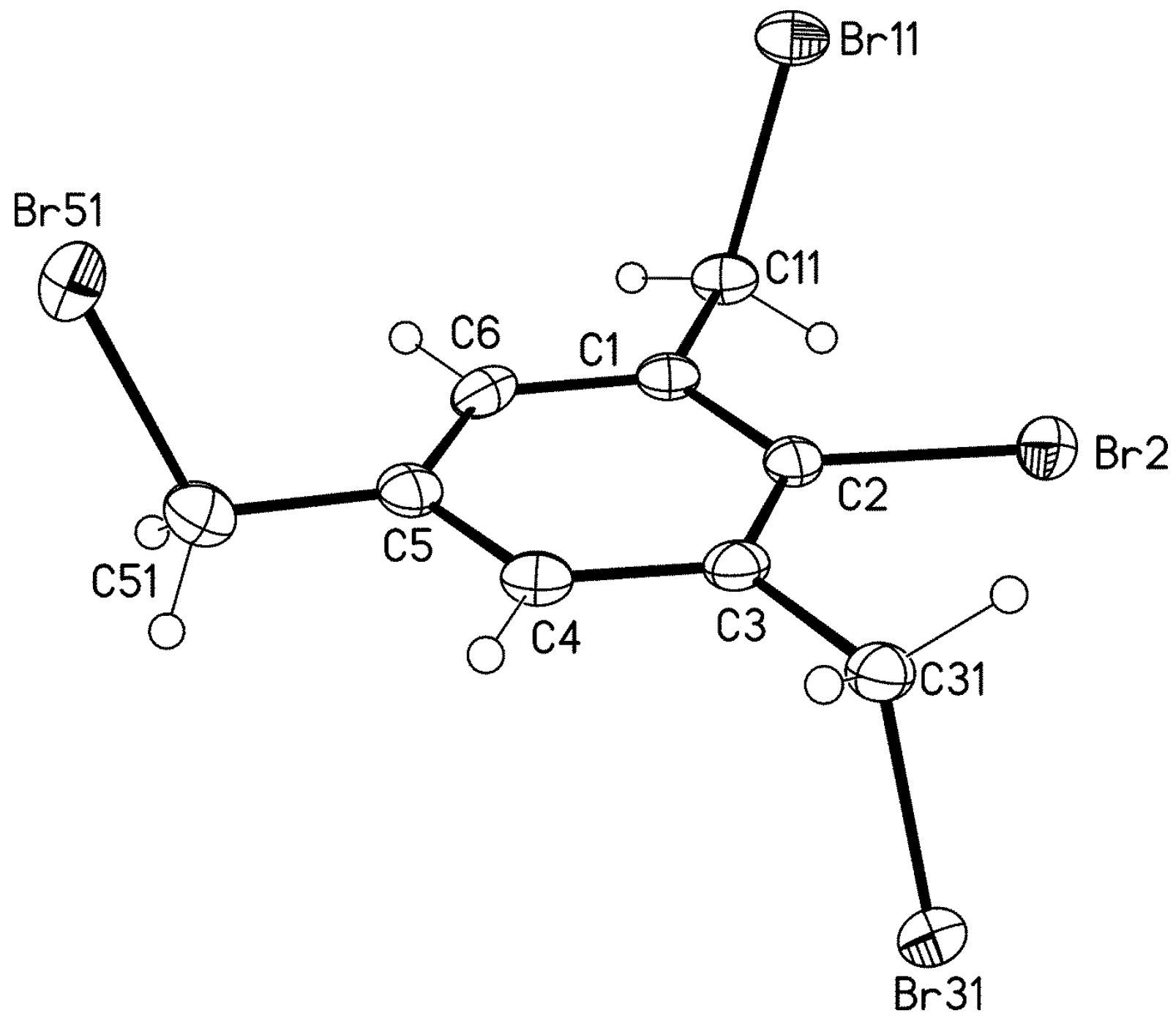

Figure S7. Molecular structure of S1 (displacement parameters are drawn at $50 \%$ probability level).

\section{References}

1. Fery-Forgues, S.; Lavabre, D., Are fluorescence Quantum yields So Tricky to Measure? A Demonstration Using Familiar Stationery Products. J. Chem. Edu. 1999, 1260-1264.

2. Vives, G.; Giansante, C.; Bofinger, R.; Raffy, G.; Del Guerzo, A.; Kauffmann, B.; Batat, P.; Jonusauskas, G.; McClenaghan, N. D., Facile functionalization of a fully fluorescent perfluorophenyl BODIPY: photostable thiol and amine conjugates. Chem. Commun. 2011, 47 (37), 10425-10427. 
3. Zhao, X.; Ng, W. Y.; Lau, K. C.; Collis, A. E. C.; Horvath, I. T., Generation of (nonafluoro-tertbutoxy)methyl ponytails for enhanced fluorous partition of aromatics and heterocycles. Phys. Chem. Chem. Phys. 2012, 14 (11), 3909-3914.

4. Szabo, D.; Mohl, J.; Balint, A.-M.; Bodor, A.; Rabai, J., Novel generation ponytails in fluorous chemistry: Syntheses of primary, secondary, and tertiary (nonafluoro-tert-butyloxy)ethyl amines. J. Fluor. Chem. 2006, 127 (11), 1496-1504.

5. Program Package for ab initio Electronic Structure Calculations, TURBOMOLE, Version 6.6; a development of University of Karlsruhe and Forschungszentrum Karlsruhe GmbH 1989-2007; Turbomole $\mathrm{GmbH}$ since 2007; http://www.turbomole.com.

6. Weigend, F.; Ahlrichs, R., Balanced basis sets of split valence, triple zeta valence and quadruple zeta valence quality for $\mathrm{H}$ to Rn: Design and assessment of accuracy. Phys. Chem. Chem. Phys. 2005, 7 (18), 3297-3305.

7. Becke, A. D., Density-Functional Thermochemistry. 3. The Role of Exact Exchange. J. Chem. Phys. 1993, 98 (7), 5648-5652.

8. Zhao, Y.; Truhlar, D. G., Density functionals with broad applicability in chemistry. Acc. Chem. Res. 2008, 41 (2), 157-167.

9. Rappoport, D.; Furche, F., Property-optimized Gaussian basis sets for molecular response calculations. J. Chem. Phys. 2010, 133 (13).

10. Klamt, A.; Schuurmann, G., COSMO - A New Approach to Dielectric Screening in Solvents with Explicit Expressions for the Screening Energy and its Gradient. J. Chem. Soc. -Perkin Trans. 2 1993, (5), 799-805.

11. Sheikbahae, M.; Said, A. A.; Wei, T. H.; Hagan, D. J.; Vanstryland, E. W., Sensitive Measurement of Optical Nonlinearities Using a Single Beam. IEEE J. Quantum Electron. 1990, 26 (4), 760-769.

12. Padilha, L. A.; Webster, S.; Hu, H.; Przhonska, O. V.; Hagan, D. J.; Van Stryland, E. W.; Bondar, M. V.; Davydenko, I. G.; Slominsky, Y. L.; Kachkovski, A. D., Excited state absorption and decay kinetics of near IR polymethine dyes. Chem. Phys. 2008, 352 (1-3), 97-105.

13. Sheldrick, G. M., A short history of SHELX. Acta Crystallog. Sec. A 2008, 64, 112-122. 